\title{
Hallazgos endoscópicos en el tubo digestivo secundarios a la ingesta de cáusticos en niños atendidos en el Departamento de Urgencias
} Endoscopic findings of the digestive tract secondary to caustic ingestion in children seen at the Emergency Department

\author{
Dr. Alejandro Barrón Balderas ${ }^{a, b}$, D. en C. Mireya Robledo Aceves ${ }^{a}$, \\ Dr. Pedro Coello Ramírez, Dra. Elizabeth García Rodríguez y \\ Dr. Javier Á. Barriga Marín ${ }^{a}$
}

\section{RESUMEN}

Antecedentes. La ingesta de sustancias cáusticas en pediatría constituye una causa frecuente de atención médica en Urgencias. El uso indiscriminado de limpiadores químicos y la facilidad de adquisición son factores determinantes para estas lesiones.

Población y métodos. Estudio descriptivo analítico. Se incluyeron niños $<16$ años, entre enero de 1998 y diciembre de 2017. Se identificó el agente químico cáustico ingerido por el niño como ácido o alcalino. Se realizó una endoscopía digestiva para tipificar el grado de quemadura. Se comparó el grado de quemadura con el tipo de cáustico; por medio de la prueba de $\mathrm{chi}^{2} \mathrm{o}$ exacta de Fisher, se consideró significativo el valor de $\mathrm{P}<0,05$.

Resultados. Ingresaron 133 niños en Urgencias por ingesta de cáusticos. El agente cáustico fue ácido en el $41 \%$ y alcalino en el $59 \%$. El agente ácido más frecuente fue el ácido muriático $(36,8 \%)$, mientras que el alcalino fue la sosa (soda) cáustica $(41,4 \%)$. La quemadura del esófago fue más frecuente en la ingesta de sosa en comparación con otros cáusticos $(p=0,001)$, mientras que la quemadura del estómago $(p=0,001)$ y del duodeno $(p=0,002)$ fue estadísticamente significativa en la ingesta de ácido muriático. El grupo de edad que más frecuentemente ingirió algún cáustico $(93,2 \%)$ fueron los menores de 5 años.

Conclusiones. El cáustico ingerido con mayor frecuencia fue un agente alcalino, que provocó quemadura esofágica, mientras que un agente ácido provocó quemaduras en el estómago y el duodeno evidenciadas por endoscopía.

Palabrasclave: cáusticos, endoscopíagastrointestinal, niño.

http:/ / dx.doi.org/10.5546/ aap.2018.409

Texto completo en inglés:

http:/ / dx.doi.org/10.5546/ aap.2018.eng.409

Financiamiento:

Ninguno.

Conflicto de intereses:

Ninguno que declarar.

Recibido: 16-4-2018

Aceptado: 11-7-2018
Cómo citar: Barrón Balderas A, Robledo Aceves M, Coello Ramírez P, García Rodríguez E, et al. Hallazgos endoscópicos en el tubo digestivo secundarios a la ingesta de cáusticos en niños atendidos en el Departamento de Urgencias. Arch Argent Pediatr 2018;116(6):409-414.

\section{INTRODUCCIÓN}

En los últimos años, la industria química ha desarrollado infinidad de compuestos utilizados en la limpieza. El fácil acceso de estos potentes limpiadores y las características intrínsecas de los niños han incrementado la incidencia de lesiones por cáusticos en menores de cinco años. ${ }^{1,2}$ Las cifras de incidencia real varían según la estructura sanitaria de cada país. Algunos reportes calculan entre 5000 y 18000 casos de ingestión cáustica cada año en Estados Unidos de América (EE. UU.). ${ }^{3,4}$ Dependiendo de la exposición, los cáusticos pueden ser la causa de quemaduras gastrointestinales, dérmicas y oculares. La gravedad del daño depende del tipo, la cantidad y la concentración de la sustancia ingerida. Las sustancias alcalinas y ácidas tienen altos efectos tóxicos. ${ }^{5} \mathrm{Un} \mathrm{pH}$ menor de dos o superior a 12 son cáusticos. ${ }^{3}$ Las sustancias alcalinas, generalmente, causan lesiones más graves por su necrosis por licuefacción. ${ }^{5}$ Para los niños con ingesta de algún cáustico, la endoscopía es una técnica confiable que permite evaluar la mucosa del tubo digestivo superior y superar el inconveniente que existe entre la escasa correlación de los síntomas y la gravedad de las lesiones. Con este procedimiento, se puede delimitar la topografía y la gravedad de las lesiones. ${ }^{6}$ Se recomienda que se realice en las primeras 24 horas después del evento, porque luego de este período, puede favorecer la 
perforación del tubo digestivo con el endoscopio. ${ }^{7}$ Las complicaciones a corto plazo de la ingesta de cáusticos incluyen la perforación y la muerte, mientras que, a largo plazo, se presenta estenosis y se ha observado un incremento en el riesgo de carcinoma esofágico. ${ }^{8}$ Nuestro objetivo fue describir las características endoscópicas del tubo digestivo de acuerdo con el agente químico ingerido en pacientes pediátricos.

\section{POBLACIÓN Y MÉTODOS}

El estudio fue realizado en el Departamento de Urgencias Pediátricas y Gastroenterología Pediátrica del Hospital Civil de Guadalajara Dr. Juan I. Menchaca. La población fue de 133 niños hospitalizados por ingesta de algún cáustico, de enero de 1998 a diciembre de 2017, con edades de un mes a 15 años y 11 meses. Se determinó la ingesta del cáustico por interrogatorio indirecto, datos clínicos y endoscópicos en el Departamento de Urgencias. Por interrogatorio a los padres, se determinó el tipo de sustancia que había ingerido el niño. En cada caso, se solicitó al familiar que llevara a la unidad hospitalaria el frasco de la sustancia ingerida para determinar el tipo de cáustico y el pH de la sustancia. En la evaluación clínica, se consideró el diagnóstico de ingesta de cáustico si el menor presentaba dolor oro faríngeo, sialorrea, edema, úlceras en la cavidad oral, disfonía, odinofagia, datos de gravedad, como dolor torácico retroesternal, vómitos, hematemesis y datos de choque hipovolémico. ${ }^{9}$ Gastroenterólogos pediatras certificados realizaron una endoscopía de fibra óptica en el tubo digestivo en las primeras 24 horas después de la ingesta, cuando el paciente se encontraba estable. En la tipificación endoscópica de las quemaduras, se utilizó la clasificación de Zargar. ${ }^{10}$ (Tabla 1). Los criterios de inclusión fueron todos los niños menores de 16 años que acudieron al Servicio de Urgencias, con antecedente de ingesta de algún cáustico que fue plenamente identificado por el personal de Urgencias y se logró determinar el pH de la sustancia. Se excluyeron los niños que ingirieron alguna sustancia cáustica que estuviera mezclada con hidrocarburos o algún otro tóxico y los niños con una exposición mayor de 48 horas de la ingesta del cáustico. Se eliminaron los niños que ingirieron el cáustico y aquellos en los que no fue posible realizar la endoscopía dentro de las 6-24 horas posteriores a la exposición y cuando el paciente se encontraba con datos de choque, datos clínicos de perforación de víscera hueca o datos de obstrucción de la vía aérea superior.

\section{Análisis estadístico}

En el análisis estadístico, se obtuvieron, en las variables cualitativas, frecuencias y porcentajes. En las variables cuantitativas, promedio y desviación estándar. Para el análisis inferencial, se dividieron las exposiciones de cáusticos en tres grupos. En los agentes alcalinos, la soda cáustica; en los ácidos, el ácido muriático, y el resto de los cáusticos se englobaron en otros cáusticos. Las diferencias de los grupos fueron determinadas por medio de $\mathrm{chi}^{2}$ o la prueba exacta de Fisher. Se definió significancia si el valor de $\mathrm{P}$ fue inferior a 0,05. Se utilizó IBM SPSS Statistics versión 21 y Microsoft Excel versión 14.4.5.

\section{Consideraciones éticas}

El consentimiento informado se obtuvo de los padres de los niños en el momento de la evaluación clínica para la realización de la endoscopía. El Consejo de Investigación Local y el Comité Ético del Hospital Civil de Guadalajara Dr. Juan I. Menchaca aprobaron el protocolo de estudio, ya que cumplía con el artículo 16, capítulo I, título segundo del Reglamento de la Ley General de Salud en Materia de Investigación para la Salud en México, en donde se protegía la confidencialidad y la privacidad del sujeto de investigación como lo establece la Declaración de Helsinki.

TABLA 1. Clasificación de Zargar para lesiones endoscópicas ${ }^{10}$

\begin{tabular}{ll}
\hline Grado & Características endoscópicas \\
\hline 0 & Mucosa normal \\
I & Edema e hiperemia de la mucosa \\
II & Friabilidad, hemorragias, erosiones, ampollas, membranas blanquecinas y úlceras superficiales \\
II a & No focal profunda o úlceras circunferenciales \\
II b & Focal profunda o úlceras circunferenciales \\
III & Áreas de múltiples ulceraciones y áreas de necrosis \\
III a & Pequeñas áreas dispersas de necrosis focal \\
III b & Necrosis amplia \\
\hline
\end{tabular}




\section{RESULTADOS}

\section{Características de la muestra}

De los 133 niños con ingesta de cáusticos, $39,1 \%$ fueron del género femenino (52 casos) y $60,9 \%$ del masculino (81 casos). El grupo de edad que más frecuentemente presentó la ingesta de algún cáustico fueron menores de cinco años, que representaban el 93,2 \% (124 casos). La media de edad fue de dos años con seis meses. Seis casos $(4,5 \%)$ se encontraban en el rango de edad de 6 a 12 años y el 2,3\% (tres casos) fueron mayores de 13 años. Los pacientes tuvieron una estancia hospitalaria de 5,4 días en promedio, con una desviación de 7,8 horas. El 10,5 \% de los niños que ingirieron cáusticos (14 casos) presentaron alguna complicación tardía. La más frecuente fue estenosis esofágica $(7,2 \%)$, seguida de úlcera gástrica (1 caso), hipoxia cerebral (1 caso) y defunción (1 caso).

\section{Exposición al agente químico y características endoscópicas del tubo digestivo}

Con respecto a la exposición a los agentes químicos, 79 casos fueron por sustancias de tipo alcalino (59\%) y 54, de tipo ácido (41\%). De los agentes alcalinos, el más frecuente fue la sosa cáustica, mientras que, en los ácidos, fue el ácido muriático (Tabla 2).

Los hallazgos endoscópicos de los niños que ingirieron cáusticos se describen en la Tabla 3. Los niños que presentaron quemadura de primer grado en la vía aérea fueron los que ingirieron ácido muriático (dos casos); en ambos casos, los familiares provocaron el vómito en el menor antes de acudir a la unidad hospitalaria y esta acción pudo provocar o incrementar la lesión, como una doble quemadura de entrada y salida. Las áreas en donde se observó la lesión fueron, principalmente, la laringe, la epiglotis y las cuerdas vocales. Se encontró que la quemadura en la cavidad oral fue más frecuente en el grupo de sosa cáustica, sin ser estadísticamente significativa. En el esófago, se reportó una $\mathrm{p}=0,001$ para sosa cáustica y una $\mathrm{p}=0,001$ 0,002 en el estómago y el duodeno con ácido muriático. Es decir, se encontró una asociación estadísticamente significativa en las lesiones

TABLA 2. Tipos de agentes químicos cáusticos ingeridos

\begin{tabular}{lcc} 
Agente & Porcentaje & Frecuencia \\
\hline Álcalis & & \\
$\quad$ Sosa & 41,4 & 55 \\
$\quad$ Hipoclorito de sodio & 8,3 & 11 \\
$\quad$ Hidróxido de potasio & 5,3 & 7 \\
$\quad$ Amoniaco & 4,5 & 6 \\
Ácidos & & \\
$\quad$ Ácido muriático & 36,8 & 49 \\
$\quad$ Ácido bórico & 1,3 & 2 \\
$\quad$ Ácido oxálico & 0,8 & 1 \\
$\quad$ Ácido sulfúrico & 0,8 & 1 \\
$\quad$ Ácido acético & 0,8 & 1 \\
Total & $\mathbf{1 0 0}$ & $\mathbf{1 3 3}$ \\
\hline
\end{tabular}

TABLA 3. Diagnóstico endoscópico de las quemaduras del tubo digestivo de acuerdo con el agente químico ingerido

\begin{tabular}{|c|c|c|c|c|c|}
\hline $\begin{array}{l}\text { Grados de quemadura por } \\
\text { evaluación endoscópica }\end{array}$ & $\begin{array}{l}\text { Álcalis } \\
\text { Sosa } \\
\text { n }(\%)\end{array}$ & $\begin{array}{c}\text { Ácido } \\
\text { Ácido muriático } \\
\mathbf{n}(\%)\end{array}$ & $\begin{array}{c}\text { Otros cáusticos } \\
\text { n (\%) }\end{array}$ & $\mathbf{P}^{\mathbf{a}}$ & $\begin{array}{l}\text { Total } \\
\text { n }(\%)\end{array}$ \\
\hline Vía aérea (grado I) & 0 & $2(100)$ & 0 & 0,11 & $2(1,5)$ \\
\hline Cavidad oral ${ }^{\mathrm{b}}$ & $31(49,2)$ & $22(34,9)$ & $10(15,9)$ & 0,66 & $63(47,3)$ \\
\hline Grado I $^{\mathrm{b}}$ & $26(46,4)$ & $20(35,7)$ & $10(17,9)$ & 0,74 & $56(42,1)$ \\
\hline Grado II a & $5(71,4)$ & $2(28,6)$ & 0 & 0,60 & $7(5,2)$ \\
\hline Esófago $^{\mathrm{b}}$ & $48(50)^{\mathrm{b}}$ & $36(37,5)$ & $12(12,5)$ & 0,001 & $96(72,1)$ \\
\hline Grado I $^{\mathrm{b}}$ & $25(51)$ & $18(36,7)$ & $6(12,3)$ & 0,001 & $49(36,8)$ \\
\hline Grado II $a^{b}$ & $12(46,1)$ & $11(42,3)$ & $3(11,6)$ & 0,02 & $26(19,5)$ \\
\hline Grado II $b^{b}$ & $10(55,5)$ & $5(27,7)$ & $3(16,8)$ & 0,005 & $18(13,5)$ \\
\hline Grado III a & $1(50)$ & $1(50)$ & 0 & 0,28 & $2(1,5)$ \\
\hline Grado III b & 0 & $1(100)$ & 0 & 0,60 & $1(0,8)$ \\
\hline Estómago & $8(32)$ & $16(64)^{b}$ & $1(4)$ & 0,001 & $25(18,7)$ \\
\hline Grado I & $6(37,5)$ & $10(62,5)$ & 0 & 0,01 & $16(12)$ \\
\hline Grado II a & $1(14,3)$ & $5(71,4)$ & $1(14,3)$ & 0,02 & $7(5,2)$ \\
\hline Grado III a & $1(100)$ & 0 & 0 & 0,92 & $1(0,8)$ \\
\hline Grado III b & 0 & $1(100)$ & 0 & 0,17 & $1(0,8)$ \\
\hline Duodeno & $1(12,5)$ & $7(87,5)^{b}$ & 0 & 0,002 & $8(6)$ \\
\hline Grado I & $1(14,2)$ & $6(85,7)$ & 0 & 0,02 & $7(5,2)$ \\
\hline Grado II a & 0 & $1(100)$ & 0 & 0,42 & $1(0,8)$ \\
\hline
\end{tabular}

a: prueba de Fisher; b: prueba de chi²; valor de $\mathrm{P}=<0,05$. 
del esófago con sosa cáustica y las lesiones en el tubo digestivo más bajo, como el estómago y el duodeno, con ácido muriático.

En la Figura 1, se ilustran cuatro fotografías del esófago y del estómago de niños atendidos en nuestro Servicio, con diferentes grados de lesión de acuerdo con la clasificación de Zargar.

\section{DISCUSIÓN}

Mientras más pequeño es el niño, más frecuentes son las lesiones dentro del hogar, como caídas, heridas e ingesta de tóxicos. Desde 1966, la Organización Mundial de la Salud (OMS) ha reportado que los países en vías de desarrollo presentan tasas elevadas de lesiones en niños menores de 5 años. ${ }^{11}$ En nuestro estudio, la edad de los niños que ingirieron algún cáustico se encontraba en un rango menor de 5 años, como lo reportado en la literatura. ${ }^{6,12-16}$ Los factores que favorecen que ocurran estos accidentes van desde la curiosidad propia de la edad, la capacidad cada vez mayor para alcanzar sitios y manipular aparatos peligrosos, el descuido de los cuidadores, la falta de áreas de recreación en los domicilios hasta el exceso de confianza de los padres respecto a la capacidad del niño para evitar accidentes. ${ }^{11}$ Es probable que estos factores, junto con la frecuencia de almacenar cáusticos en frascos de bebidas o alimentos sin el etiquetado adecuado, favorezcan este tipo de accidentes. ${ }^{16-19}$ Desafortunadamente, no existe una fórmula o una guía exacta para evitar que los menores estén en contacto con estos químicos. Nuutinen, en su estudio, refiere que la ingesta de cáusticos disminuyó significativamente en Finlandia desde 1969 debido al control y uso limitado en la venta de estos productos. ${ }^{20}$ En países como China, se ha abogado, por largo tiempo, por el uso de contenedores para productos de limpieza a prueba de niños para disminuir la incidencia de estas lesiones. ${ }^{13}$ Sin embargo, en EE.UU., un país que ha adoptado estas medidas de control y seguridad en los contenedores, en un análisis de 1990 a 2006, se ha observado que las edades de ingesta de cáusticos eran similares a las reportadas en la literatura, menores de 5 años, con un pico máximo de 1 a 3 años. Se observó que los productos adecuadamente etiquetados, sobre todo, en botellas de spray, incrementaron de $30 \%$ a $40 \%$ como causa de intoxicación o quemaduras en los niños. Es decir, los niños que tuvieron algún contacto tóxico con estos productos fueron los mismos que manipularon el químico en forma frecuente o que referían haber visto a sus padres usarlos. ${ }^{6}$ Esta característica apoya la hipótesis de que la edad curiosa del menor $y$, principalmente,
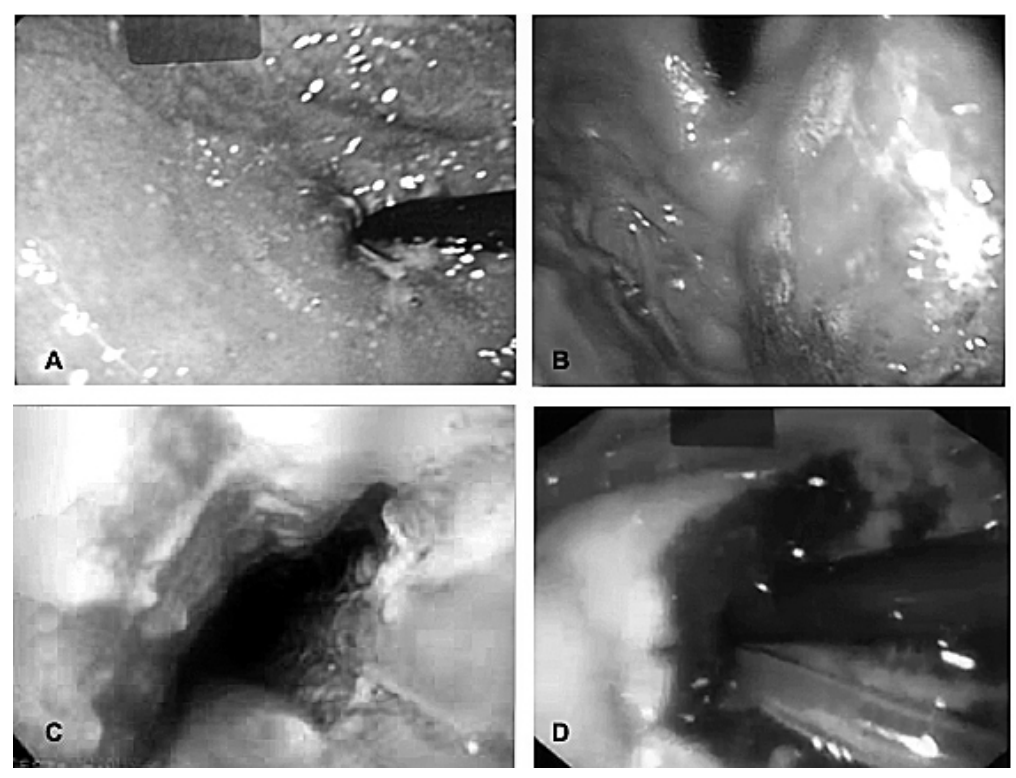

A) Eritema en el fundus gástrico, lesión de grado I. B) Ampollas, membranas blanquecinas y úlceras superficiales en el fundus gástrico no focales profundas, lesión de grado II a. C) Úlceras circunferenciales en el tercio medio del esófago, lesión de grado II b. D) Áreas de necrosis dispersas en el fundus gástrico, lesión de grado III a 
el descuido del cuidador son la principal causa de estos accidentes. El papel del pediatra en países en vías de desarrollo en la prevención de la ingesta de cáusticos no se limita solo a informar a los padres de poner los productos fuera del alcance del menor, sino de fomentar la legislación para evitar la venta libre de estos productos en frascos no apropiados y sin etiquetado.

Johnson, en 2012, publicó el impacto en la salud pública de la ingesta de cáusticos en EE. UU., con un gasto de 28860 dólares en promedio por caso, con una estancia hospitalaria de 4,13 días en promedio. ${ }^{7}$ En nuestro estudio, no se evaluó el gasto por caso, ya que el servicio de salud en nuestro país era financiado por el Gobierno dentro del Programa de Seguro Popular, pero sí se analizó el tiempo hospitalario, con un promedio de 5,4 días, similar a lo observado por Johnson. Temiz, en 2012, en Turquía, observó que los pacientes con quemaduras del tubo digestivo de grado I y II a tenían estancias hospitalarias con un promedio de cinco días, similar a lo observado en el estudio de Johnson y en este, pero, si el grado de quemadura era de III a o III $b$, las estancias hospitalarias se incrementaban hasta 15 días en promedio por las complicaciones. ${ }^{14}$ Aunque aquí no se estadificó la estancia hospitalaria por el grado endoscópico de la quemadura, la mayoría de nuestros niños presentaron grados de I a IIb y menos del $5 \%$ (5 casos) presentaron grados IIIa o IIIb en el esófago y el estómago; probablemente por esta razón, nuestras estancia hospitalaria fue menor.

En un metaanálisis de 64 artículos publicado en 2016, observaron que la ingesta de cáusticos con respecto al género fue más frecuente en los varones, con promedios de $42 \%$ (intervalo de confianza-IC-del $95 \%$ :0,29-0,57). Sin embargo, esta diferencia no fue estadísticamente significativa, ${ }^{21}$ similar a lo observado en el nuestro, en que hubo una ligera tendencia hacia el género masculino, pero no se encontró como un factor de riesgo. Este mismo estudio reportó que las sustancias más frecuentemente ingeridas en los estudios analizados fueron alcalinos. La sosa cáustica fue el agente causal más frecuente, seguida del ácido muriático. ${ }^{21}$ En nuestro estudio, la sosa fue el producto más ingerido en 55 casos (un producto alcalino), seguido por el ácido muriático en 49 casos. Yanowsky, en su estudio descriptivo realizado también en nuestra ciudad, reportó que, en el $82,8 \%$ de los niños que ingirieron cáustico, fue sosa líquida, seguida por el ácido muriático, ${ }^{12}$ la misma tendencia que en el nuestro, lo que evidencia un claro problema de salud en el país. En México, no existe una legislación para limitar la concentración de los productos de limpieza, principalmente, en pequeños comercios, donde se venden estos productos a granel, sin etiquetado y sin contenedores seguros.

En la evaluación endoscópica del tubo digestivo, se encontró que la mayoría de las lesiones fueron de grado I, IIa y IIb en la ingesta de sosa, en la que el $20 \%$ de estos niños (11 casos) presentaron estenosis esofágica como complicación tardía, similar a lo que reportaron Sánchez y colaboradores en 2010. ${ }^{22}$ Riffat, en 2009, reportó que el $50 \%$ de los pacientes con lesiones de grado II, subsecuentemente, desarrollaban estenosis esofágica. La sosa líquida no tiene un sabor fuerte lo que favorece que su ingesta sea mayor, a diferencia de las sustancias ácidas, que son más amargas. Además, su consistencia acuosa hace que se adhieran a los puntos de estrechamiento del esófago, como la cricofaríngea, el estrechamiento a nivel del bronquio izquierdo principal y el esfínter de esófago inferior, lo que causa lesiones más profundas en estas zonas por la licuefacción de la mucosa, la necrosis y la penetración hasta el músculo. ${ }^{5,23}$ En nuestros niños que ingirieron ácido muriático, durante la evaluación endoscópica del tubo digestivo, se pudieron observar lesiones de grado I y II a tanto en el estómago como en el duodeno. Temiz, en 2012, reportó que la tasa de lesiones en el estómago fue más elevada, estadísticamente significativa, en el grupo de niños que ingirieron ácidos, en comparación con los que ingirieron sustancias alcalinas, como se observó aquí.

Las sustancias ácidas causan lesiones de coagulación, que limita la penetración profunda en los tejidos. Por su baja viscosidad, estas sustancias transitan rápidamente al estómago. ${ }^{14}$ Estos factores favorecen que los ácidos provoquen lesiones en los niveles más bajos del tubo digestivo, como el duodeno.

La presentación clínica de los niños con ingesta de cáusticos, como sialorrea, dolor en la cavidad oral y vómito, así como las úlceras en la cavidad oral, no es predictora de lesiones más graves. La evaluación endoscópica dentro de las primeras 24 horas después del evento es la técnica más efectiva para establecer la gravedad de la lesión y puede ser pronóstico para la presentación de complicaciones tardías, principalmente, de esófago. $7,13-15,18,21,23,24$

La debilidad de nuestro estudio es que no se 
logró determinar, por medio del interrogatorio, la cantidad de la sustancia química que ingirió el niño, lo que podría ayudar a explicar las características de las lesiones que se observaron a través de la endoscopía.

\section{CONCLUSIÓN}

Los químicos alcalinos provocan lesiones en el tubo digestivo alto y en el esófago, mientras que los ácidos lesionan las porciones más bajas, como el duodeno, debido a sus características químicas.

\section{REFERENCIAS}

1. Montoya MA. Productos químicos de uso común en el hogar. En Toxicología clínica. México: Méndez Editores; 2002.Págs.187-94.

2. Gallegos HA. Intoxicación por cáusticos. En Córdoba D. Toxicología. 4. ${ }^{\text {ta }}$ ed. Bogotá: Manual Moderno; 2001.Págs.76472.

3. Bautista Casanovas A, Argüelles Martin F. Ingesta de cáusticos. En SEGHNP-AEP. Protocolos diagnósticosterapéuticos de gastroenterología hepatología y nutrición pediátrica. Madrid: Ergón; 2010.Págs.121-30.

4. Alba-Rodríguez M, Meza-Flores JL. Características clínicoepidemiológicas en pacientes con ingesta de cáusticos en el Hospital Hipólito Unanue. Rev Gastroenterol. 2003; 23(2):115-25.

5. AriciMA, OzdemirD, Oray NC, etal. Evaluacion of caustics and household detergents exposure in an emergency service. Hum Exp Toxicol. 2012; 31(6):533-8.

6. McKenzie LB, Ahir N, Stolz U, Nelson NG. Household cleaning product-related ijuries treated in US emergency departments in 1990-2006. Pediatrics. 2010; 126(3): 509-16.

7. Johnson CM, Brigger MT. The public health impact of pediatric caustic ingestion injuries. Arch Otolaryngol Head Neck Surg. 2012; 138(12):1111-5.

8. Lupa M, Magne J, Guarisco L, Amedee R. Update on the diagnosis and treatment of caustic ingestion. Ochsner J. 2009; 9(2):54-9.

9. Diagnóstico, manejo inicial y criterios quirúrgicos de urgencia en quemadura de esófago por cáusticos. México DF: Instituto Mexicano del Seguro Social;2013. [Consulta: 12 de julio de 2018]. Disponible en: www.imss.gob.mx/sites/ all/statics/guiasclinicas/659GER.pdf.
10. Zargar SA, Kochhar R, Mehta S, Mehta SK. The role of fiberoptic endoscopy in the management of corrosive ingestion and modified endoscopic classification of burns. Gastrointest Endosc. 1991; 37(2): 165-9.

11. Torres-Márquez M, Fonseca Pelegrín CL, Díaz-Martínez $\mathrm{MD}$, et al. Accidentes en la infancia: una problemática actual en pediatría. Medisan. 2010; 14(3):368-78.

12. Yanowsky-Reyes G, Aguirre-JáureguiO, Trujillo-PonceSA, et al. Ingesta de sustancias químicas en esófago, análisis de las complicaciones tempranas y tardías, en el manejo de los mismos; una revisión de 70 casos. Arch Med. 2013; 9(1):1-8.

13. Chen TY, Ko SF, Chuang JH, et al. Predictors of esophageal stricture in children with unintentional ingestion of caustic agents. Chang Gung Med J. 2003; 26(4):233-9.

14. Temiz A, OguzkurtP, EzerSS, etal.Predictability of outcome of caustic ingestion by esophagogastroduodenoscopy in children. World J Gastroenterol. 2012; 18(10):1098-103.

15. Losada M, Rubio M, BlancaJA, Pérez C. Ingesta de cáusticos en niños, experiencia de 3 años. Rev Chil Pediatr. 2015; 86(3):189-93.

16. Weldon E, Martye PM. Caustic soda poisoning in Ghana - an alarming increase. Paediatr Int Child Health. 2012; 32(3):158-60.

17. Sevilla-Francisco E, Larios-ArceoF, Navarro-Peña JM, Celis A. Costo anual del tratamiento por quemaduras esofágicas en pacientes pediátricos. Rev Med IMSS. 2004; 42(2):137-43.

18. Kucuk G, Gollu G, Ates U, et al. Evaluación de lesiones esofágicas secundarias a la ingesta de sustancias cáusticas no rotuladas: serie de casos pediátricos. Arch Argent Pediatr. 2017; 115(2):e85-8.

19. KurowskiJA, Kay M. Causticingestions and foreign bodies ingestions in pediatric patients. Pediatr Clin North Am. 2017; 64(3):507-24.

20. Nuutinen M, Uhari M, Karvali T, Kouvalainen K. Consequences of causticingestions in children. Acta Paediatr. 1994; 83(11):1200-5.

21. Rafeey M, Ghojazadeh M, Sheikhi S, Vahedi L. Caustic ingestion in children: a systematic review and metaanalysis. J Caring Sci. 2016; 5(3):251-65.

22. Sánchez-Ramírez CA, Larrosa-Haro A, Vásquez-Garibay EM, Larios-Arceo F. Caustic ingestion and oesophageal damage in children: clinical spectrum and feeding practices. J Paediatr Child Health. 2011; 47(6):378-80.

23. Riffat F, Cheng A. Pediatric causticingestion: 50 consecutive case and a review of the literature. Dis Esophagus. 2009; 22(1):89-94.

24. Rodríguez-Reynosa LL. Ingestión de sustancias cáusticas: reporte de un caso y revisión de la literatura. Rev Gastrohnup. 2010; 12(1):s4-9. 